\title{
Expansion Velocity of Ejecta in Tycho's Supernova Remnant Measured by Doppler Broadened X-ray Line Emission
}

\author{
Asami Hayato ${ }^{1,2}$, Hiroya Yamaguchi ${ }^{1}$, Toru Tamagawa ${ }^{1}$, Satoru Katsuda ${ }^{2}$, Una Hwang ${ }^{2}$, \\ John P. Hughes ${ }^{3}$, Midori Ozawa ${ }^{4}$, Aya Bamba ${ }^{5,6}$, Kenzo Kinugasa ${ }^{7}$, Yukikatsu Terada ${ }^{8}$, Akihiro \\ Furuzawa $^{9}$, \\ Hideyo Kunieda ${ }^{9}$, and Kazuo Makishima ${ }^{10,1}$ \\ hayato@crab.riken.jp
}

\begin{abstract}
We show that the expansion of ejecta in Tycho's supernova remnant (SNR) is consistent with a spherically symmetric shell, based on Suzaku measurements of the Doppler broadened X-ray emission lines. All the strong $\mathrm{K} \alpha$ line emission show broader widths at the center than at the rim, while the centroid energies are constant across the remnant (except for Ca). This is the pattern expected for Doppler broadening due to expansion of the SNR ejecta in a spherical shell. To determine the expansion velocities of the ejecta, we applied a model for each emission line feature having two Gaussian components separately representing red- and blue-shifted gas, and inferred the Doppler velocity difference between these two components directly from the fitted centroid energy difference. Taking into account the effect of projecting a three-dimensional shell to the plane of the detector, we derived average spherical expansion velocities independently
\end{abstract}

\footnotetext{
${ }^{1}$ RIKEN, 2-1 Hirosawa, Wako, Saitama 351-0198, Japan

${ }^{2}$ NASA Goddard Space Flight Center, Greenbelt, MD 20771, USA

${ }^{3}$ Department of Physics and Astronomy, Rutgers University, 136 Frelinghuysen Road, Piscataway, NJ 08854-8019, USA

${ }^{4}$ Department of Physics, Graduate School of Science, Kyoto University, Kita-Shirakawa, Sakyo-ku, Kyoto 6068502, Japan

${ }^{5}$ School of Cosmic Physics, Dublin Institute for Advanced Studies 31 Fitzwilliam Place, Dublin 2, Ireland

${ }^{6}$ Institute of Space and Astronautical Science, Japan Aerospace Exploration Agency, 3-1-1 Yoshinodai, Tyuo-ku, Sagamihara, Kanagawa 252-5210, Japan

${ }^{7}$ Gunma Astronomical Observatory, 6860-86, Nakayama, Takayama-mura, Agatsuma-gun, Gunma 377-0702, Japan

${ }^{8}$ Department of Physics, Saitama University, Shimo-Okubo 255, Sakura, Saitama 338-8570, Japan

${ }^{9}$ Division of Particle and Astrophysical Science, Graduate School of Science, Nagoya University, Furo-cho, Nagoya 464-8602, Japan

${ }^{10}$ Department of Physics, The University of Tokyo, 7-3-1 Hongo, Bunkyo-ku, Tokyo 113-0033, Japan
} 
for the $\mathrm{K} \alpha$ emission of $\mathrm{Si}, \mathrm{S}, \mathrm{Ar}$, and $\mathrm{Fe}$, and $\mathrm{K} \beta$ of Si. We found that the expansion velocities of $\mathrm{Si}, \mathrm{S}$, and Ar ejecta of $4700 \pm 100 \mathrm{~km} \mathrm{~s}^{-1}$ are distinctly higher than that obtained from $\mathrm{Fe} \mathrm{K} \alpha$ emission, $4000 \pm 300 \mathrm{~km} \mathrm{~s}^{-1}$, which is consistent with segregation of the $\mathrm{Fe}$ in the inner ejecta. Combining the observed ejecta velocities with the ejecta proper-motion measurements by Chandra, we derived a distance to the Tycho's SNR of $4 \pm 1 \mathrm{kpc}$.

Subject headings: supernova remnants - supernovae: general - X-rays: individual (Tycho's SNR)

\section{Introduction}

Type Ia supernovae ( $\mathrm{SNe}$ ), the thermonuclear explosions of accreting $\mathrm{C}+\mathrm{O}$ white dwarfs, are important for their role as standard candles to measure the distance to their host galaxies, and also as the main sources for the production of the Fe-group elements. However, our understanding of the physical mechanism of the explosion, as well as the details of the progenitor systems, is incomplete. The kinetic energy distribution of the ejecta holds important information about the propagation of the supernova burning front and the resulting nucleosynthesis. In sufficiently young supernova remnants (SNRs), this information is retained to some degree and can be probed by studying the $\mathrm{X}$-ray emission of the shocked ejecta.

Tycho's SNR is the remnant of the Galactic SN recorded by Tycho Brahe in 1572, and is securely classified as a Type Ia SNR based on the observed light curve (Baade 1945; Ruiz-Lapuente 2004) and the light-echo spectrum (Krause et al. 2008). As the prototypical Ia SNR, Tycho's SNR has been targeted for study of the explosion mechanism. Badenes et al. (2006) made a comparison between spatially integrated X-ray spectra of Tycho's SNR taken by XMM-Newton and Chandra and synthetic X-ray spectra based on several different Type Ia explosion models. They concluded that the observed spectra are well reproduced by a one- dimensional delayed detonation model with some degree of chemical stratification, with Fe-peak elements interior to intermediate-mass elements (IMEs, i.e., Si, S, Ar, Ca).

The narrow band images of Tycho's SNR obtained by $A S C A$ show that the Fe-K $\alpha$ emission is actually located interior to the emission lines of IMEs and Fe-L emission (Hwang \& Gotthelf 1997). Furthermore, Hwang et al. (1998) found that the Fe-K emitting ejecta have a higher electron temperature and a lower ionization age than the IME ejecta. These results imply that the Fe-K emitting ejecta are situated more inside of the remnant and has been heated by the reverse shock more recently than the other ejecta. Observations with Chandra (Warren et al. 2005) and XMMNewton (Decourchelle et al. 2001) confirm the spectral segregation between the IME and Fe. The same situation has been reported for other Ia SNRs such as SN 1006 (Yamaguchi et al. 2008) and LMC SNRs 0509-67.5 and 0519-69 (Kosenko et al. 2008, 2010). 
Thanks to the the spectral capability of Suzaku, Furuzawa et al. (2009) discovered a significant broadening of $\mathrm{Fe} \mathrm{K} \alpha$ line at the center of the Tycho remnant. They concluded that the shell of Fe $\mathrm{K} \alpha$ emitting ejecta is expanding with a line-of-sight velocity of $2800-3350 \mathrm{~km} \mathrm{~s}^{-1}$. They also inferred line broadening for He-like $\mathrm{Si}$ and $\mathrm{S} \mathrm{K} \alpha$ emission, but did not compute quantitative velocities. In this paper, we investigate the three-dimensional structure of the ejecta in Tycho's SNR and determine the expansion velocities.

\section{Observations and Data Reduction}

Suzaku observations of Tycho's SNR and an off-source background were carried out on 2006 June 26-29 and 29-30, respectively, as a part of the Scientific Working Group observing time. The pointing position for the SNR was $(\mathrm{RA}, \mathrm{Dec})=\left(00^{\mathrm{h}} 25^{\mathrm{m}} 20^{\mathrm{s}}, 64^{\circ} 08^{\prime} 18^{\prime \prime}\right)$; that for the background position was $\left(00^{\mathrm{h}} 36^{\mathrm{m}} 54^{\mathrm{s}}, 64^{\circ} 17^{\prime} 42^{\prime \prime}\right), 1^{\circ} .27$ offset from the SNR along the Galactic plane.

Suzaku carries two active instruments: four X-ray Imaging Spectrometers (XIS: Koyama et al. 2007) placed at the focal planes of four X-ray Telescopes (XRT: Serlemitsos et al. 2007) and a non-imaging Hard X-ray Detector (HXD: Takahashi et al. 2007). Each XIS has a $17 .^{\prime} 8 \times 17 .^{\prime} 8$ field of view with a half-power diameter for the XRT of $\sim 2^{\prime}$. One of the four XIS sensors (XIS 1 ) is a back-illuminated CCD with high sensitivity at $<1 \mathrm{keV}$, while the others (XIS 0,2 , and 3 ) are front-illuminated (FI) CCDs with high efficiency and low background at $>5 \mathrm{keV}$. All of the XIS sensors were operated in the normal full-frame clocking mode without any spaced-raw charge injection (SCI: Uchiyama et al. 2009) for both the SNR and background observations 1 .

The HXD data sanalysis were already reported by Tamagawa et al. (2009), so we present here the detailed analysis of the XIS data. We focus on only the FI sensors (XIS 0, 2, 3), because these have better calibration of the energy gain near the Si-edge. We reprocessed the revision 2.0.6.13 data products using the xispi software (HEASOFT version 6.5) with the version 20080825 makepi file. After the reprocessing, the data were cleaned with standard screening criteria for the cleaned event data of the XIS. The effective exposures of the SNR and background were $101 \mathrm{ks}$ and $51 \mathrm{ks}$, respectively.

For the spectral fitting, we used response matrices created with xisrmfgen software version 2007-05-14 using the version $20080311 \mathrm{rmf}$ param file. We estimated the accuracy of the XIS energy scale for our own data and response functions using the line centroid energies of the ${ }^{55} \mathrm{Fe}$ calibration sources at two of the four corners of each XIS chip. We fitted Mn K $\alpha$ lines from ${ }^{55} \mathrm{Fe}$ with Gaussian models and found that the line centroids of XIS 0,2 , and 3 were all $0.1-0.14 \%$ higher than

\footnotetext{
${ }^{1}$ Tycho's SNR was observed again by Suzaku on 2008 August 4-8 and 11-12 as one of the large proposal programs of the third announcement of opportunity observing cycle, this time with SCI on. However, we report only the analysis of the 2006 data in this paper because the current calibration of the response for the SCI-off data is better than for the SCI-on data (Ozawa et al. 2009).
} 
the expected energy of $5.985 \mathrm{keV}$. This is somewhat better than the systematic uncertainty of $\pm 0.2 \%$ reported by Ozawa et al. (2009). We also checked the width of the $\mathrm{Mn} \mathrm{K} \alpha$ line is fully consistent with the expected instrumental broadening, even with all three FI sensors merged. The response files were generated by the xissimarfgen software, assuming a homogeneous disk-like radiation source with a radius of $4^{\prime}$ corresponding to the azimuthally averaged radius of Tycho's SNR measured from the Chandra image.

\section{Overall Features}

A three-color XIS FI image of the SNR is shown in Figure 1, Red, green, and blue colors

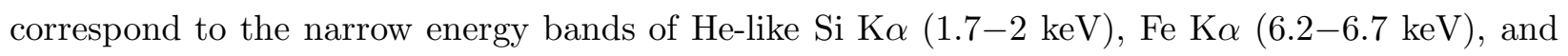
the hard continuum band $(7-13 \mathrm{keV})$. The images of the $\mathrm{Si}$ and $\mathrm{Fe} \mathrm{K} \alpha$ emission, which represent the ejecta distribution, are brighter in north and fainter in south, while the hard band image is brightest in the southwest. These agree with the trends seen in more detailed Chandra and XMM-Newton images (Hwang et al. 2002; Warren et al. 2005; Badenes et al. 2006).

The background-subtracted XIS spectrum of the entire SNR is shown in Figure 2. The source spectrum was taken from a circle with a radius of 4.65, with data from all three FI sensors merged to improve photon statistics. The background spectrum was extracted from a $7^{\prime}$-radius circular region on the off-source observation. In the spectrum, we can identify prominent K-shell emission line features of the He- and also some $\mathrm{H}$-like ions of $\mathrm{Mg}$, $\mathrm{Si}, \mathrm{S}, \mathrm{Ar}$, and $\mathrm{Ca}$, as well as emission from less ionized Cr, Mn and Fe (Tamagawa et al. 2009).

\section{Spatially-Resolved Spectra}

Figure 4 in Furuzawa et al. (2009) clearly shows that the K $\alpha$ line blends of Si, S, and Fe are broadened at the center of the remnant, relative to the rim. However, their quantitative study was limited to the Fe $\mathrm{K} \alpha$ emission. Therefore, we extend their analysis to the lines of IMEs. We divided Tycho's SNR into 4 radial regions as shown in Figure 1, with a circle at the center and three-quarter circular rings numbered from 1 (inner) to 4 (outer). The radius of the inner region 1 was $1 ! 41$, and the thickness of the surrounding outer regions was 1 '.08. We excluded the southeast quadrant $\left(60^{\circ}-150^{\circ}\right.$ where the angles increase counterclockwise from north) because of the presence

of irregular ejecta clumps (Vancura et al. 1995; Decourchelle et al. 2001). We adopted the same background spectrum as used by 93 . Since the spectrum at energies below $1.7 \mathrm{keV}$ is dominated by the Fe L- shell emission, where the atomic physics is complicated, we focused only on the energy band above $1.7 \mathrm{keV}$ in the following sections. For simplicity, we divided the spectrum into two energy bands: the $1.7-5 \mathrm{keV}$ band for the lines of IMEs (\$4.1), and the $5-8 \mathrm{keV}$ band for the Fe-K lines $(\$ 4.2)$. 


\section{1. $\quad 1.7-5 \mathrm{keV}$ Spectra}

To examine the radial changes of emission features, we fitted spectra taken from each region with a phenomenological model featuring an absorbed power-law for the continuum emission plus twenty Gaussian components for the line emission. The column density for the foreground interstellar gas was set to be $N_{\mathrm{H}}=7 \times 10^{21} \mathrm{~cm}^{-2}$ following Cassam-Chenaï et al. (2007). The Gaussians represented transitions for the elements $\mathrm{Si}, \mathrm{S}, \mathrm{Ar}$, and $\mathrm{Ca}$ for He-like and H-like ions: $\operatorname{He} \alpha(n=2 \rightarrow n=1), \operatorname{He} \beta\left(1 s 3 p \rightarrow 1 s^{2}\right)$, He $\gamma\left(1 s 4 p \rightarrow 1 s^{2}\right)$ in the He-like ions, and Ly $\alpha(2 p \rightarrow 1 s)$, Ly $\beta(3 p \rightarrow 1 s)$ in the H-like ions. We modeled the He $\alpha$ transitions by a single Gaussian, which actually contains a blend of the forbidden, inter-combination, and resonance lines. For example, in $\mathrm{Si} \mathrm{He} \alpha$ blend, the rest energies of the constituent lines are $1839.4 \mathrm{eV}$ (forbidden), $1853.7 \mathrm{eV}$ (inter-combination), and $1864.9 \mathrm{eV}$ (resonance), with the blending giving an extra effective width of about $15 \mathrm{eV}$ (Hwang \& Gotthelf 1997). We also represented each blend of He $\beta$ and its surrounding satellite lines by a single Gaussian model. We note that the effective width for $\mathrm{Si} \operatorname{He} \beta$ is comparable to that of $\mathrm{He} \alpha$, based on the non-equilibrium ionization (NEI) model version 1.1 in XSPEC. The continuum spectra must be a combination of thermal and non-thermal emission, but these contributions are difficult to estimate separately (e.g., Tamagawa et al. 2009). We thus checked that the subsequent results for the Gaussian components do not change significantly even if we use a bremsstrahlung model for the continuum instead of a power-law model.

The line centroid, width, and intensity of the prominent He $\alpha$ blends of Si, S, Ar, and Ca, as well as the $\mathrm{He} \beta$ blends of $\mathrm{Si}$ and $\mathrm{S}$, were fitted freely. The other emission lines are too weak to constrain the Gaussian parameters. Thus, we fixed the energy difference between the prominent line features above and weaker line features of the same element; for example, the energy difference between S He $\alpha$ and S Ly $\alpha$ was fixed to $178 \mathrm{eV}$. Here we assumed electron temperature $k T_{e}$ of $\sim$ $1 \mathrm{keV}$ and ionization timescale $n_{e} t$ of $\sim 10^{11} \mathrm{~cm}^{-3} \mathrm{~s}$, typical in Tycho's SNR (Hwang et al. 1998), because the centroid energy of the He $\alpha$ blend depends on $k T_{e}$ and $n_{e} t$; and used the Astrophysical Plasma Emission Database (APED: Smith et al. 2001). The line widths were linked to each other for lines of the same element (e.g., S He $\alpha, \mathrm{S} \operatorname{Ly} \alpha, \mathrm{S} \mathrm{He} \beta, \mathrm{S} \mathrm{He} \gamma$, and $\mathrm{S} \operatorname{Ly} \beta$ ). The intensities of the prominent He-like emission above and the relatively strong Ly $\alpha$ lines of Si and S, were freely fitted. For lines other than those, we fixed the intensity ratios: we took $\operatorname{He} \gamma / \operatorname{He} \beta=0.3$ and $\operatorname{Ly} \beta / \operatorname{Ly} \alpha=0.1$ for the plasma in Tycho's SNR $\left(k T_{e}=1 \mathrm{keV}\right.$ and $n_{e} t=10^{11} \mathrm{~cm}^{-3} \mathrm{~s}$, as noted above). These intensity ratios do not vary much with $n_{e} t$ and vary only $10 \%$ over a decade in $k T_{e}$; hence the uncertainties here are negligibly small. We also fixed the ratios of $\mathrm{Ly} \alpha / \mathrm{He} \alpha$ and $\mathrm{He} \beta / \mathrm{He} \alpha$ for Ar and Ca to be the same as these of $\mathrm{S}$.

With these models and assumptions, we fitted all the four spectra. The reduced $\chi^{2}$ values and degrees of freedom (dof) in regions 1, 2, 3, and 4 were 1.37 (791), 1.42 (724), 1.74 (858), and 1.56 (846), respectively. The large reduced $\chi^{2}$ values are attributed to the high statistics of spectra and our approximations above for the fitting model. In addition, there might be some contaminating emission from the less ionized ions (e.g., Li-like). We thus conclude that the best-fit models do reproduce the spectra sufficiently well. Individual components of the best-fit models for regions 1 
and 4 are shown in Figure 3 (a-1) and (b-1), respectively. The best-fit parameters of all the fits are listed in Table 1.

\section{2. $\quad 5-8 \mathrm{keV}$ Spectra}

We also fitted the $5-8 \mathrm{keV}$ band spectra with a power-law for the continuum, plus three Gaussian components for $\mathrm{Cr}$ and $\mathrm{Fe} \mathrm{K} \alpha$, and $\mathrm{Fe} \mathrm{K} \beta$ line blends. Since $\mathrm{Cr} \mathrm{K} \alpha$ and $\mathrm{Fe} \mathrm{K} \beta$ emission are very weak, these widths were tied to that of $\mathrm{Fe} \mathrm{K} \alpha$. The other parameters were allowed to vary freely. The reduced $\chi^{2}$ s and dof for regions 1, 2, 3 and 4 were 1.25 (115), 1.22 (214), 1.07 (335), and 0.86 (298), respectively. All the fits for the four spectra from regions 1-4 were acceptable. The 5-8 keV spectra from regions 1 and 4 with the best-fit models are shown in Figure 3 (a-2) and (b-2), respectively. The best-fit parameters are listed in Table 1 .

\section{Radial Line Profiles}

Figure 4 shows the centroid energies, widths, and intensities of the $\mathrm{K} \alpha$ line blends of $\mathrm{Si}, \mathrm{S}$, $\mathrm{Ar}, \mathrm{Ca}$ and $\mathrm{Fe}$, obtained in the previous section. We found that the centroid energies of all the elements except for $\mathrm{Ca}$ are constant with respect to radius within the systematic uncertainties of

$\pm 0.2 \%$, indicated in each left box. This systematic error was estimated by Ota et al. (2007) using the Suzaku observations of extended sources with Fe K $\alpha$ emission (Cygnus Loop and Sagittarius C), and represents the gain variation on the same CCD chip at the $90 \%$ confidence level. In contrast to the constancy of the centroid energies, the widths of all the emission features decrease significantly from the center to the rim. The difference between the $\mathrm{Si}$ and Fe widths of regions 1 and 4 is $10.5 \pm 0.3 \mathrm{eV}$ and $33 \pm 6 \mathrm{eV}$, respectively.

The radial intensity profile of IMEs emission are similar to each other with a peak radius of $3^{\prime}-4^{\prime}$, while $\mathrm{Fe}-\mathrm{K} \alpha$ emission has a radius of $\sim 3^{\prime}$ which is somewhat smaller than that of the IMEs. These trends agree with the previous observations (e.g., Hwang \& Gotthelf 1997).

There is some indication of spectral variations within our source regions in Figure 1, so we investigated the azimuthal variations of the line centroids and widths by dividing regions 2, 3, and 4 evenly into three azimuthal sectors. We applied the same fitting procedure to the spectrum accumulated from each divided region. There is some variation amongst the azimuthally divided sectors, with the azimuthal variations of line centroids being $\pm 3 \mathrm{eV}$ for $\mathrm{Si}, \pm 5 \mathrm{eV}$ for $\mathrm{S}, \pm 8 \mathrm{eV}$ for $\mathrm{Ar}, \pm 20 \mathrm{eV}$ for $\mathrm{Ca}$, and $\pm 10 \mathrm{eV}$ for Fe. These values are much smaller than the corresponding line widths; however, in all the radial sectors, the widths show the same trends of being wider in the inside compared to the rim, and the centroids being more constant. The qualitative trends appear to be the same as for the azimuthally averaged spectral regions, therefore we proceed with our azimuthally averaged analysis. 
In the following subsections, we consider two simple interpretations of the radial properties of centroid energies and widths as discussed by Furuzawa et al. (2009): (a) plasma characterized by multiple ionization ages, and (b) Doppler shifts resulting from expansion in a shell.

\subsection{Plasma with Multiple Ionization Ages}

It is expected that the plasma in the SNR will have multiple ionization ages. Since the reverse shock propagates from the outside of the remnant inward, the shocked ejecta will have different $n_{e} t$ between the outside (near the contact discontinuity) and the inside (where the reverse shock has just passed through), with the more recently shocked ejecta generally having a lower

$n_{e} t$ and a different $k T_{e}$ from the outer ejecta, with details depending on the ejecta density profile (Dwarkadas \& Chevalier 1998).

Plasma with a range of $n_{e} t$ values easily makes the observed line blend broaden at the center of the remnant, since line centroids strongly depend on $n_{e} t$. For example, based on the NEI model of version 1.1, the centroid of the $\mathrm{K} \alpha$ line blend of $\mathrm{S}$ at $k T_{e}=1 \mathrm{keV}$ varies from $2.41 \mathrm{keV}$ to 2.45 $\mathrm{keV}$ depending on the $n_{e} t$ values of $10^{10}-10^{11} \mathrm{~cm}^{-3} \mathrm{~s}$, which may result in a line broadening of $\sim 40 \mathrm{eV}$. However, our results show that the centroid energies are constant across the remnant within $0.2 \%$. This implies that the plasma emitting each line or blend has the same average $n_{e} t$ all over the SNR. Therefore, a multiple $n_{e} t$ plasma does not provide a fully satisfying explanation of our observation.

\subsection{Doppler Shift by the Shell Expansion}

Another case we consider is an expanding shell of ejecta. The emission lines from retreating and approaching gas are red- and blue- shifted, respectively. Thus, the line emission at the projected center of the remnant is expected to be broadened. At the rim, on the other hand, a narrow line would be observed, since there is only a small line-of-sight component of the velocity. In addition, if the expansion is spherically symmetric (and the plasma is reasonably uniform), the centroid energies will be constant with radius. We found that the observed line profiles are well reproduced by the Doppler broadening of the spherically symmetric shell expansion.

\subsection{Properties of Ca He-like $\mathrm{K} \alpha$ line}

As shown in Figure 4, the Ca He $\alpha$ blend is the only feature where the centroid energy is seen to gradually increases from the center to the rim. Possibly, this suggests that the Ca ejecta might be in multiple ionization ages unlike the other ejecta. However, the Ca He $\alpha$ blend is heavily contaminated by $\mathrm{Ar} \mathrm{He} \beta$, and thus the inferred properties of the Ca emission depends rather 
strongly on the assumed intensity ratio, $\mathrm{He} \beta / \mathrm{He} \alpha$ of Ar. A future observation with higher energy resolution is required to reliably investigate the accurate properties of $\mathrm{Ca} \mathrm{He} \alpha$ blend.

\section{Velocities of Ejecta}

In this section, we quantitatively derive the expansion velocity with the $\mathrm{K} \alpha$ emission of $\mathrm{Si}, \mathrm{S}$, $\mathrm{Ar}$, and $\mathrm{Fe}$, and $\mathrm{K} \beta$ of $\mathrm{Si}$, assuming that the ejecta are expanding in a spherical shell.

\subsection{Method of Velocity Determination}

In a spherically symmetric shell expansion, a broadened line should have both red- and blueshifted components. If we apply such a two-Gaussian model (with red- and blue-shifted components) to a single broadened line, the line energy shift (i.e., relative to the rest frame) gives the Doppler velocity as

$$
\frac{\left|E_{\mathrm{obs}, i}-E_{0}\right|}{E_{0}}=\frac{v_{\perp i}}{c}
$$

where $E_{\mathrm{obs}, i}$ and $v_{\perp i}$ are the observed centroid energy of the red- or blue-shifted line and the line-of-sight velocity in each region $i(i=1-4)$, respectively, and $E_{0}$ is the line energy at the rest frame. We introduce here the energy shift in each region $i, \delta E_{i}=\left|E_{\mathrm{obs}, i}-E_{0}\right|$. Assuming front-back velocity symmetry, the parameter we measure is $2 \times \delta E_{i}$, corresponding to the energy difference between the red- and blue-shifted lines.

Once we obtain $v_{\perp i}$, we can convert it to the expansion velocity $v_{\exp }$ by considering two important effects: the projection of the three-dimensional shell onto the plane of the detector, and the limited spatial resolution of the Suzaku XRT. In an appendix, we calculate the factors $C_{i}$ which

represent what percentage of the $v_{\exp }$ would be observed as $v_{\perp i}$ in each region $i$. In other words, $v_{\exp }$ can be expressed in terms of $C_{i}$ and the observable parameter $v_{\perp i}$ as

$$
v_{\exp }=\frac{v_{\perp i}}{C_{i}} .
$$

The calculated $C_{i}$ factors are summarized in Table 2,

\subsection{Spectral Fitting}

As a first step, we derived the expansion velocities by fitting the spectra of regions 1 and 4 at the same time, because the difference between $v_{\perp 1}$ and $v_{\perp 4}$ is expected to be the largest.

To test for spherically symmetric expansion, we therefore fitted the $1.7-5 \mathrm{keV}$ spectra with the model of an absorbed power-law for the continuum and Gaussian components for emission lines. The column density for the foreground interstellar gas was set as in 4.1 . We applied Gaussian 
models including red- and blue-shifted components, for each line feature. We left the centroid energies of the both lines free, while the widths and intensities were set to be equal to each other. Because we fitted the spectra of regions 1 and 4 at the same time, we actually applied two pairs of the red- and blue-shifted lines (four Gaussians in total) for each line feature. According to Equation 1, the ratio of $\delta E_{1} / \delta E_{4}$ equals the ratio of $v_{\perp 1} / v_{\perp 4}$. Since $v_{\exp }$ should be the same for regions 1 and 4, Equation 2 gives $\delta E_{1} / \delta E_{4}=C_{1} / C_{4}$. In our fitting, we therefore fixed the ratio of $\delta E_{1} / \delta E_{4}=C_{1} / C_{4}$. As a result, $v_{\exp }$, the width, and intensity were the only three parameters that were allowed to vary freely for each set of four Gaussians.

We employed twenty sets of four Gaussians for the observed line features: $\operatorname{He} \alpha, \operatorname{Ly} \alpha, \operatorname{He} \beta$, $\mathrm{He} \gamma, \mathrm{Ly} \beta$ of $\mathrm{Si}, \mathrm{S}, \mathrm{Ar}$, and Ca. We derived the $v_{\exp }$ independently for the prominent He $\alpha$ blends of $\mathrm{Si}, \mathrm{S}$, and $\mathrm{Ar}$, and $\mathrm{He} \beta$ of $\mathrm{Si}$. The energy separation between these and other line features were fixed as the model in 4.1 , for example, the energy difference between $\operatorname{Ar} \operatorname{He} \alpha$ and $\operatorname{Ar} \operatorname{He} \beta$ was fixed to $209 \mathrm{eV}$ (see Table 1). The sole exception is $\mathrm{S} \mathrm{Ly} \alpha$, whose energy was free in 4.1 , here, we fixed the energy shift from $\mathrm{S} \mathrm{He} \beta$ to $181 \mathrm{eV}$ to compensate for limited photon statistics. A single line width was fitted for lines of the same element (e.g., for S: $\operatorname{He} \alpha$, Ly $\alpha$, $\operatorname{He} \beta$, He $\gamma$, and Ly $\beta$ ). The exception was $\mathrm{Si} \mathrm{He} \alpha$, whose width was fitted independently from that of other Si lines, due to uncertainties in the response near the Si-edge. As noted in 4.1 , the width of the Gaussian applied to the He $\alpha$ blend includes a contribution from the blending of the constituent triplet lines $(\sim 15$ $\mathrm{eV}$ ). This broadening varies within a few $\mathrm{eV}$ depending on the plasma condition, but we checked that the measurement of the line centroid is little affected by the uncertainty of the width. The intensity ratios of weak lines to prominent lines (e.g., S Ly $\alpha / \mathrm{He} \alpha$ ) were fixed by assuming a plasma with $k T_{e} \sim 1 \mathrm{keV}$ and $n_{e} t \sim 10^{11} \mathrm{~cm}^{-3} \mathrm{~s}$, as in 4.1 . The exception here is that $\mathrm{S}$ Ly $\alpha / \mathrm{He} \alpha$ was fixed to the value obtained from the fits in $\$ 4.1$ (Table 1).

We also fitted the $5-8 \mathrm{keV}$ spectra of regions 1 and 4 using the same procedure as above. The model consisted of a power-law continuum and red- and blue-shifted Fe $\mathrm{K} \alpha$ lines. We also included the $\mathrm{Cr} \mathrm{K} \alpha$ and $\mathrm{Fe} \mathrm{K} \beta$ blends, but as single Gaussians due to limited photon statistics. We fixed the ratio of $\delta E_{1} / \delta E_{4}$ to $C_{1} / C_{4}$ following Table 2. The widths and intensities of the red- and blue-shifted lines in regions 1 and 4 were all linked to each other. Figure 5 shows the individual components of the best-fit models. The reduced $\chi^{2} \mathrm{~s}$ and dof were 1.47 (1430) for $1.7-5 \mathrm{keV}$ and 0.96 (415) for $5-8 \mathrm{keV}$ band spectra.

As a second step, we have also fitted all the spectra of regions 1, 2, 3, and 4 at the same time in exactly the same manner just described. Here our aim was to verify that the model was valid for all four regions, and also to improve the photon statistics. The ratios of energy difference among regions 1,2,3 and 4 were fixed as $\delta E_{1}: \delta E_{2}: \delta E_{3}: \delta E_{4}=C_{1}: C_{2}: C_{3}: C_{4}$. The parameters obtained from the fits are consistent with those obtained for regions 1 and 4 , and within the systematical errors. The reduced $\chi^{2}$ s and dof were 1.55 (3028) and 1.06 (970) for $1.7-5 \mathrm{keV}$ and 5-8 keV band spectra, respectively. The best fit parameters are summarized in Table 3 , 


\subsection{Expansion Velocities}

We translate $\delta E_{i}$ into $v_{\perp i}$ using Equation 1, and then convert to $v_{\exp }$ using Equation 2 and $C_{i}$ (Table 21). The derived velocities are summarized in Table 3, We note that the $v_{\text {exp }}$ of the Fe K $\alpha$ emission in Table 3 does not correspond to the line-of-sight velocity measured in Furuzawa et al. (2009), because the $v_{\text {exp }}$ represents the expansion velocity in which the projection effect and PSF effect have properly been taken into account. Meanwhile, the $v_{\perp 1}$ is the value should correspond to the line-of-sight velocity measured in Furuzawa et al. $(2009), 3040_{-210}^{+310} \mathrm{~km} \mathrm{~s}^{-1}$. Our measurement of $v_{\perp 1}$ and the line-of-sight velocity measured in Furuzawa et al. (2009) agree well each other. The

$v_{\text {exp }}$ derived from Si He $\alpha$ and $\mathrm{He} \beta$ are consistent with each other. Therefore, we conclude that the systematic uncertainty of the Si-edge in the response is small enough to determine the velocity. The expansion velocity obtained from the $\mathrm{He} \alpha$ blends of $\mathrm{Si}, \mathrm{S}$, and Ar are clearly higher than that obtained from the $\mathrm{Fe} \mathrm{K} \alpha$ blends.

\section{Discussion}

Based on the analysis of spatially resolved spectra, we have demonstrated that (1) the ejecta shell is expanding in a generally spherical and symmetric manner, and (2) the expansion velocities of the IME ejecta are significantly higher than that of the Fe $\mathrm{K} \alpha$ emitting ejecta. These results then allow us to discuss the distance to Tycho's SNR, the ejecta segregation, and the reverse shock velocity.

\subsection{Distance to Tycho's SNR}

Using Chandra high-resolution images of Tycho's SNR obtained in 2000, 2003, and 2007, Katsuda et al. (2009) measured the expansion rates of both the forward shock and the reverse shocked ejecta. They found that the mean proper-motion of the Si-rich layer is $\mu \sim 00^{\prime \prime} 25 \mathrm{yr}^{-1}$. This is consistent with the result Hughes (2000) had derived using ROSAT data. Combining with our expansion velocities of $4700 \pm 100 \mathrm{~km} \mathrm{~s}^{-1}$ (Table 3), we obtained a range of the distance to the SNR of $D=(4.0 \pm 1.0)\left(v / 4700 \mathrm{~km} \mathrm{~s}^{-1}\right)\left(\mu / 0^{\prime \prime} 25 \mathrm{yr}^{-1}\right)^{-1} \mathrm{kpc}$. The relatively large uncertainty is mainly due to the azimuthal variation of the proper motion of the ejecta. Our result is the first estimate of the distance to Tycho derived solely from X-ray observations.

The distances inferred by previous studies is shown in Figure 6. The distance of around 2-3 kpc estimated by modeling the observed $\mathrm{H} \alpha$ line spectra (green in Figure 6) has been most widely

cited thus far, but is model-dependent. Krause et al. (2008) recently derived a larger distance of $3.8_{-1.1}^{+1.5} \mathrm{kpc}$, based on the SN peak luminosity estimated by the observed optical light-echo spectrum. Our estimate is consistent with the result from Krause et al. (2008). 


\subsection{Ejecta Segregation}

The Fe-K radial profile peaks at $\sim 180^{\prime \prime}$ which is a distinctly smaller radius than the IME and Fe-L emission of 200" (Decourchelle et al. 2001; Warren et al. 2005). In addition, the Fe-K emitting ejecta has about 100 times lower $n_{e} t$ than the IMEs and Fe-L emitter $\left(10^{11} \mathrm{~cm} \mathrm{~s}^{-1}\right.$ : Hwang et al. 1998). These results suggest that the plasma emitting Fe-K has been heated by the reverse shock more recently than the other materials. Since the reverse shock propagates from the outside toward the center, the Fe-K and $\mathrm{Si}$ emission are tracers of the interior and exterior material in the SNR, respectively.

Our results show that the Si ejecta also have higher $v_{\exp }\left(4700 \pm 100 \mathrm{~km} \mathrm{~s}^{-1}\right)$ compared to the Fe K $\alpha$ emitting ejecta $\left(4000 \pm 300 \mathrm{~km} \mathrm{~s}^{-1}\right)$. These velocity measurements add to the morphological and spectral evidence that the Fe-K emitting ejecta are segregated interior to the Si ejecta. The expansion rate ratio of $\mathrm{Fe} \mathrm{K} \alpha$ emission to Si ejecta is derived to be $(4000 / 180) /(4700 / 200) \sim 0.93$.

\subsection{Reverse Shock Velocity}

The angular radius of the reverse shock of $183^{\prime \prime}$ estimated by Warren et al. (2005) corresponds to $r_{\mathrm{rs}}=3.5 \pm 0.9 \mathrm{pc}$ at a distance of $4.0 \pm 1.0 \mathrm{kpc}$. Given the age of $434 \mathrm{yr}$, the velocity of unshocked ejecta $v_{\text {un,ej }}$ is then estimated to be $8000 \pm 2000 \mathrm{~km} \mathrm{~s}^{-1}$ at a radius of $3.5 \mathrm{pc}$. The post-shock velocity $v_{\text {sh,ej }}$ can be approximated as the obtained expansion velocity of $\mathrm{Fe}, 4000 \pm 300$ $\mathrm{km} \mathrm{s}^{-1}$. Under the assumption of strong shock, conservation laws for mass, momentum, and energy across the shock front yield the following Rankine-Hugoniot relation,

$$
v_{\mathrm{un}, \mathrm{ej}}-v_{\mathrm{rs}}=\left(\frac{\gamma+1}{\gamma-1}\right)\left(v_{\mathrm{sh}, \mathrm{ej}}-v_{\mathrm{rs}}\right),
$$

where $v_{\mathrm{rs}}$ and $\gamma$ are the reverse shock velocity in the observer's frame and specific heat ratio, respectively. Since Warren et al. (2005) argued that the reverse shock of Tycho's SNR is not strongly accelerating cosmic-rays (unlike the forward shock), we here take $\gamma=5 / 3$ as appropriate for a non-relativistic ideal gas. Equation 3 then gives $v_{\mathrm{rs}}=2700 \pm 800 \mathrm{~km} \mathrm{~s}^{-1}$, and hence the upstream velocity in the shock-rest frame is obtained to be $\bar{v}_{\mathrm{rs}}=v_{\mathrm{un}, \mathrm{ej}}-v_{\mathrm{rs}}=5300 \pm 2100 \mathrm{~km} \mathrm{~s}^{-1}$.

This value allows us to estimate the explosion energy of Tycho's SN by comparing with a self-similar model for the evolution of young SNRs interacting with a uniform density ISM. In Figure 2d of Dwarkadas \& Chevalier (1998), $\bar{v}_{\mathrm{rs}}$ is expected to be $3.4 \times 10^{3} E_{51}^{1 / 2}\left(M_{\mathrm{ej}} / 1.4 M_{\odot}\right)^{-1 / 2}$ $\mathrm{km} \mathrm{s}^{-1}$ throughout most of the SNR's evolution, for either $r^{-7}$ power-law and exponential density distributions of the ejecta, where $E_{51}$ is the explosion energy in units of $10^{51}$ ergs. Thus, assuming an ejecta mass of $1.4 M_{\odot}$, the explosion energy of Tycho's SN is derived to be $(2.5 \pm 2.0) \times 10^{51}$ ergs. Given that the error is large, the derived energy range includes the standard value for normal Type Ia SNe.

From the Rankine-Hugoniot relations, the post-shock temperature for each particle species $a$ 
is given as

$$
k T_{a}=\frac{2(\gamma-1)}{(\gamma+1)^{2}} m_{a} \bar{v}_{\mathrm{rs}}^{2}=\frac{3}{16} m_{a} \bar{v}_{\mathrm{rs}}^{2}
$$

where $m_{a}$ is the particle mass. Given $\bar{v}_{\text {rs }}=5300 \pm 2100 \mathrm{~km} \mathrm{~s}^{-1}$, the temperature of the shocked Fe is expected to be $k T_{\mathrm{Fe}}=3 \pm 2 \mathrm{MeV}$. Such a high temperature would result in a line broadening of $\Delta E=70 \pm 30 \mathrm{eV}$ due to the thermal Doppler effect. We measured the width of the Fe-K blend of $55 \pm 3 \mathrm{eV}$ (Table 3), where an intrinsic line broadening of $20-30 \mathrm{eV}$ is expected for the plasma in the range of $k T_{e}>1.6 \mathrm{keV}$ and $10<\log n_{e} t \mathrm{~cm}^{-3} \mathrm{~s}<10.7$ (Hwang et al. 1998; Furuzawa et al. 2009). The additional width of $\sim 50 \mathrm{eV}$ which cannot be explained by the intrinsic line broadening might be explained by the thermal Doppler broadening of Fe ions. Future missions with high energy resolution, like Astro- $H$, will help us to accurately measure ion temperatures and study the heating process at the reverse shock of young SNRs in detail.

\section{Summary}

We analyzed the X-ray spectra of Tycho's SNR using the Suzaku 100 ks observation. We summarize our results below:

1. We obtained the radial dependence of centroid energies, widths, and intensities for the $\mathrm{K} \alpha$ emission of $\mathrm{Si}, \mathrm{S}, \mathrm{Ar}, \mathrm{Ca}$, and $\mathrm{Fe}$ for annular regions covering nearly three-fourths of the azimuth around the SNR. The centroid energies of all line blends except $\mathrm{Ca}$ are constant with radius, while the widths significantly become narrower from the center to the rim. We found that the observed line properties are well explained by a spherically symmetric shell expansion of the ejecta.

2. We derived the expansion velocities of ejecta with Doppler broadened K $\alpha$ emission of $\mathrm{Si}, \mathrm{S}, \mathrm{Ar}$ and $\mathrm{Fe}$, and $\mathrm{K} \beta$ of Si independently. We fitted each broadened line feature with two Gaussians representing red- and blue-shifted gas, and obtained the expansion velocity from the centroid energy separation between two lines. We found that expansion velocities measured for $\mathrm{K} \alpha$ emission of $\mathrm{Si}, 4700 \pm 100 \mathrm{~km} \mathrm{~s}^{-1}$, are clearly higher than that measured for Fe $\mathrm{K} \alpha, 4000 \pm 300$ $\mathrm{km} \mathrm{s}^{-1}$.

3. Combining the obtained expansion velocity with the proper-motion measurement by Chandra, the distance to Tycho's SNR is estimated to be $4 \pm 1 \mathrm{kpc}$.

To determine the true expansion velocity $v_{\text {exp }}$, we have to calculate the correction factors $C_{i}$ introduced in Equation 2. $C_{i}$ describes the projection effect of the three-dimensional shell onto the detector plane, including the photon smearing effects caused by the limited spatial resolution of the Suzaku XRT. In this appendix, we calculate the correction factors $C_{i}$. 
First we define the regions that we use in the following calculations. As shown in Figure 7 , we divide the entire SNR into ten "SKY" regions. The region enclosed by the innermost circle is named SKY 1, the next two annular regions are SKY 2 and 3, and the outer six annular regions, in which a quarter region is excluded, are named SKY 4 (inner) to SKY 9 (outer). The radii of the SKY regions are $30^{\prime \prime}$ for SKY 1, and are incremented by $30^{\prime \prime}$ toward the outer edge of the SNR. The quarter region in the southeast is named SKY 0. The regions $1-4$ are the detector regions used in 4 (Figure 1). The regions 1, 2, 3, and 4 roughly correspond to the SKY 1-3, 4-5, 6-7, and $8-9$, respectively.

\section{A. Projection Effects}

Consider a thin and spherically symmetric shell with radius $r_{\text {sh }}$. The shell is expanding with velocity $v_{\exp }$. The polar angle $\theta$ is defined as the angle measured from the horizontal line which intersects the center of the spherical shell, and the azimuth angle $\phi$ represents an angle around the horizontal line. The sky region $j(j=0,1, \ldots, 9)$ is described by using the range of $\theta\left(\theta_{1}^{j}\right.$ and $\left.\theta_{2}^{j}\right)$ and $\phi$. The $\theta_{1}^{j}$ and $\theta_{2}^{j}$ angles are given as

$$
\begin{aligned}
& \theta_{1}^{0}=\arcsin \left(r_{3} / r_{\mathrm{sh}}\right), \theta_{2}^{0}=\arcsin \left(r_{9} / r_{\mathrm{sh}}\right), \\
& \theta_{1}^{j}=\arcsin \left(r_{j-1} / r_{\mathrm{sh}}\right), \theta_{2}^{j}=\arcsin \left(r_{j} / r_{\mathrm{sh}}\right)(j=1,2, \ldots, 9),
\end{aligned}
$$

where the radius $r_{k}$ is given as

$$
r_{k}=k \cdot 30^{\prime \prime}(k=0,1, \ldots, 9) .
$$

We here consider only the half of the spherical shell $(0<\theta<90 \mathrm{deg})$ in to evaluate $\delta E_{i}$.

The projected velocities $v_{j}$ of the SKY region $j$ are found as the mean line-of-sight velocity $v_{\perp}=v_{\exp } \cos \theta$. By integrating $v_{\perp}$ over the solid angle, the mean velocity $v_{j}$ is described as

$$
v_{j}=v_{\exp } \cdot A_{j},
$$

where

$$
A_{j}=\frac{\int_{\theta_{1}^{l}}^{\theta_{2}^{j}} \cos \theta \sin \theta d \theta}{\int_{\theta_{1}^{j}}^{\theta_{2}^{j}} \sin \theta d \theta}
$$

Since $\theta_{1}^{j}$ and $\theta_{2}^{j}$ are functions of $r_{\mathrm{sh}}, A_{j}$ also depends on $r_{\mathrm{sh}}$. We calculated $A_{j}$ for the radius $r_{\mathrm{sh}}$ at 2 arcsec intervals.

The actual ejecta shell has a finite width in radius $r_{\mathrm{sh}}$. Decourchelle et al. (2001) and Warren et al. (2005) reported $r_{\mathrm{sh}}=190^{\prime \prime}-220^{\prime \prime}$ for $\mathrm{Si}$, and $r_{\mathrm{sh}}=180^{\prime \prime}-200^{\prime \prime}$ for Fe. We therefore find an average 
of $A_{j}$ over the given range of $r_{\mathrm{sh}}$ as

$$
\bar{A}_{j}=\frac{\sum_{r_{\mathrm{sh}}=r_{\mathrm{sh}}^{\min }}^{r_{\mathrm{sh}}^{\max }} A_{j}\left(r_{\mathrm{sh}}\right)}{\sum_{r_{\mathrm{sh}}=r_{\mathrm{sh}}^{\min }}^{r_{\mathrm{sh}}^{\max }} 1} .
$$

In Table 4, we summarize the calculated $\bar{A}_{j}$.

\section{B. Limited spatial resolution of XRT}

We estimate the smearing effect caused by the limited spatial resolution of the XRT. We use the xissim software, which simulates interactions of X-ray photons incident upon the XRT/XIS system using ray-tracing and Monte Carlo techniques (Ishisaki et al. 2007).

First, we prepare the input image files for the simulation. Because the distributions of $\mathrm{K}$ shell emission for IMEs and Fe are slightly different (e.g., Hwang \& Gotthelf 1997), we prepare two narrow-band Chandra images: the Si and S $(1.8-1.9$ plus $2.4-2.5 \mathrm{keV})$ band, and the Fe-K $(6-7$ $\mathrm{keV}$ ) band. Next, we divide each Chandra image into the SKY regions introduced in Figure 7 , and we take this divided image as an input to the xissim simulator. In the simulation, we fix the input photon energy to be monochromatic: $1.8 \mathrm{keV}$ and $6.5 \mathrm{keV}$ for $\mathrm{Si}+\mathrm{S}$ and Fe, respectively. Finally, in the simulated images, we accumulate the number of photons in each detector region $1-4$. We repeat this procedure for both energy bands $(\mathrm{Si}+\mathrm{S}$ and $\mathrm{Fe}$ ) and for all ten different $\mathrm{SKY}$ regions (SKY 0-9).

Results of the simulations are summarized in Table 5. We introduce the smearing factor $F_{i j}$, which represents the fraction of the number of photons that originate in a certain SKY region $j$ $(j=0-9)$ relative to to the number of photons detected in a certain detector region $i(i=1-4)$. For example, in the Fe $\mathrm{K}$ band, $F_{13}=0.194$ means that $19.4 \%$ of photons detected in region 1 originate from SKY 3.

\section{Correction Factors}

We derive the correction factor $C_{i}$ by multiplying the projection factor $\bar{A}_{j}$ (Table 4) by the smearing factor $F_{i j}$ (Table 5) as

$$
C_{i}=\sum_{j=0}^{9} F_{i j} \bar{A}_{j}(i=1,2,3,4) .
$$

The derived values are summarized in Table 2 , 
We gratefully acknowledge all members of the Suzaku hardware and software teams and the Science Working Group. A.H. is Research Fellow of Japan Society for the Promotion of Science (JSPS). S.K. is supported by JSPS Postdoctoral Fellowships for Research Abroad. J.P.H. was supported by NASA grant NNG05GP87G.

\section{REFERENCES}

Badde, W., 1945, ApJ, 102, 309

Badenes, C., et al. 2006, ApJ, 645, 1373

Cassam-Chenaï, G. et al. 2007, ApJ, 665, 315

Decourchelle, A., et al. 2001, A\&A, 365, L218

Dwarkadas, V. V., \& Chevalier, R. A. 1998, ApJ, 497, 807

Furuzawa, A., et al. 2009, ApJ, 693, L61

Ghavamian, P. et al. 2001, ApJ, 547, 995

Hughes, J. P. 2000, ApJ, 545, L53

Hwang, U., \& Gotthelf, E.V. 1997, ApJ, 475, 665

Hwang, U., et al. 1998, ApJ, 497, 833

Hwang, U., et al. 2002, ApJ, 581, 110

Ishisaki, Y. et al. 2007, PASJ, 59, 113

Katsuda et al. 2010, ApJ, 709, 1387

Kosenko, D., et al. 2008, A\&A, 490, 223

Kosenko, D., et al. 2010, eprint arXiv:1001.0983

Koyama, K., et al. 2007, PASJ, 59, S23

Krause, O., et al. 2008, Nature, 456, 617

Ota et al. 2007, PASJ, 59, S351

Ozawa, M., et al. 2009, PASJ, 61, S1

Ruiz-Lapuente, P. 2004, ApJ, 612, 357

Ruiz-Lapuente, P., et al. 2004, Nature, 612, 357 
Schwarz, U. J., et al. 1980, MNRAS, 192, 67

Schwarz, U. J., et al. 1995, A\&A, 229, 193S

Serlemitsos, P. J. 2007, PASJ, 59, S9

Smith, R. C., et al. 1991, ApJ, 375, 652S

Smith, R. K., et al. 2001, ApJ, 556 L91

Takahashi, T. et al. 2007, PASJ, 59, S35

Tamagawa, T., et al. 2008, PASJ, 61, S167

Uchiyama, H., et al. 2009, PASJ, 61, S9

Vancura, O., Gorenstein, P., \& Hughes, J. P. 1995, ApJ, 441, 680

Völk, H. J., et al. 2008, A\&A, 483, 529

Warren, J. S., et al. 2005, ApJ, 634, 376

Yamaguchi, H., et al. 2008, PASJ, 60, S141 


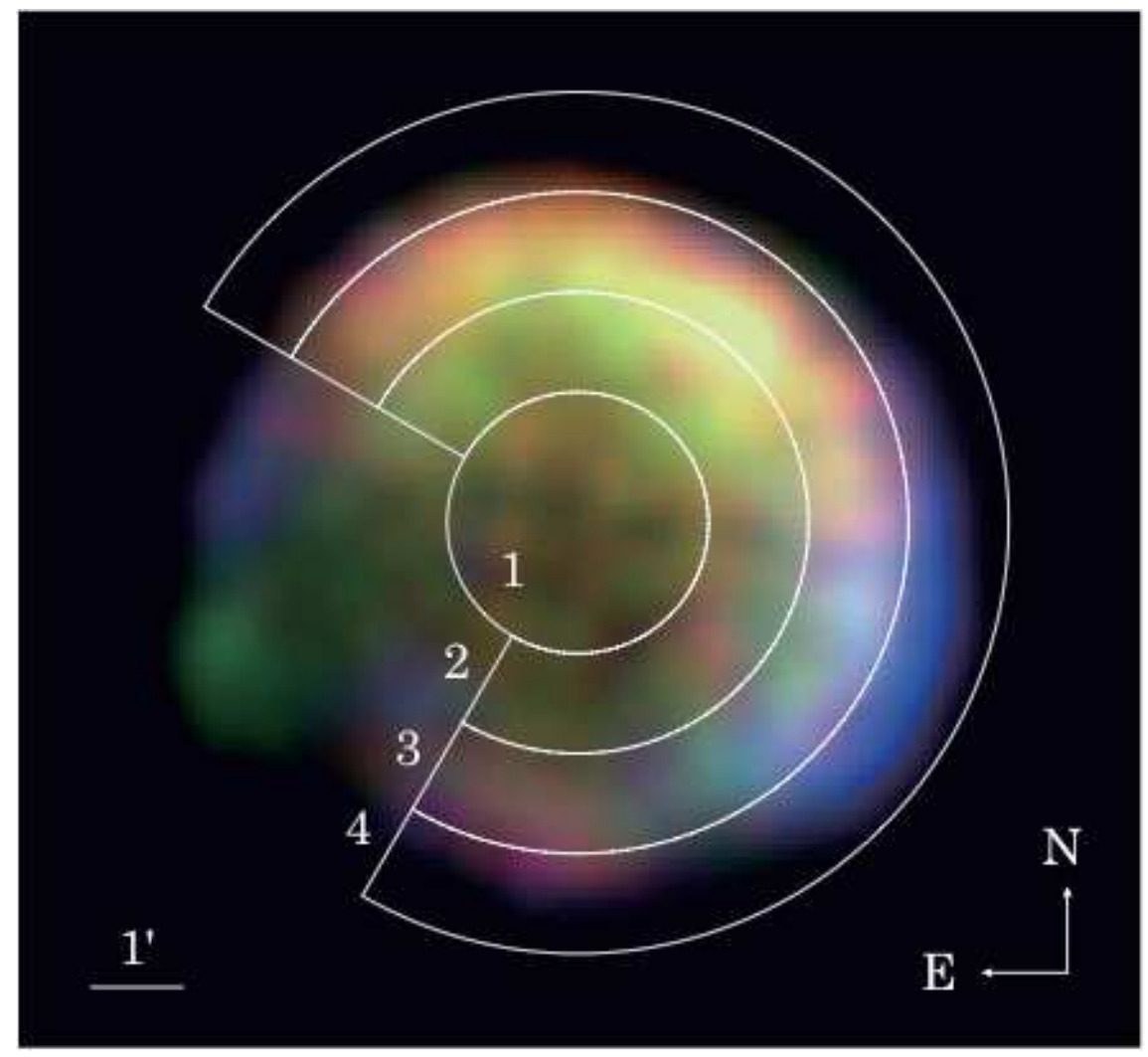

Fig. 1. - Three color XIS FI (XIS 0+2+3) image of Tycho's SNR. Red, green, and blue correspond to the energy bands of $\mathrm{Si} \mathrm{He} \alpha(1.7-2 \mathrm{keV})$, the Fe $\mathrm{K} \alpha(6.2-6.7 \mathrm{keV})$, and the hard band energy band $(7-13 \mathrm{keV})$, respectively. The white circle and quarter sectors are the regions where we extract spectra. 


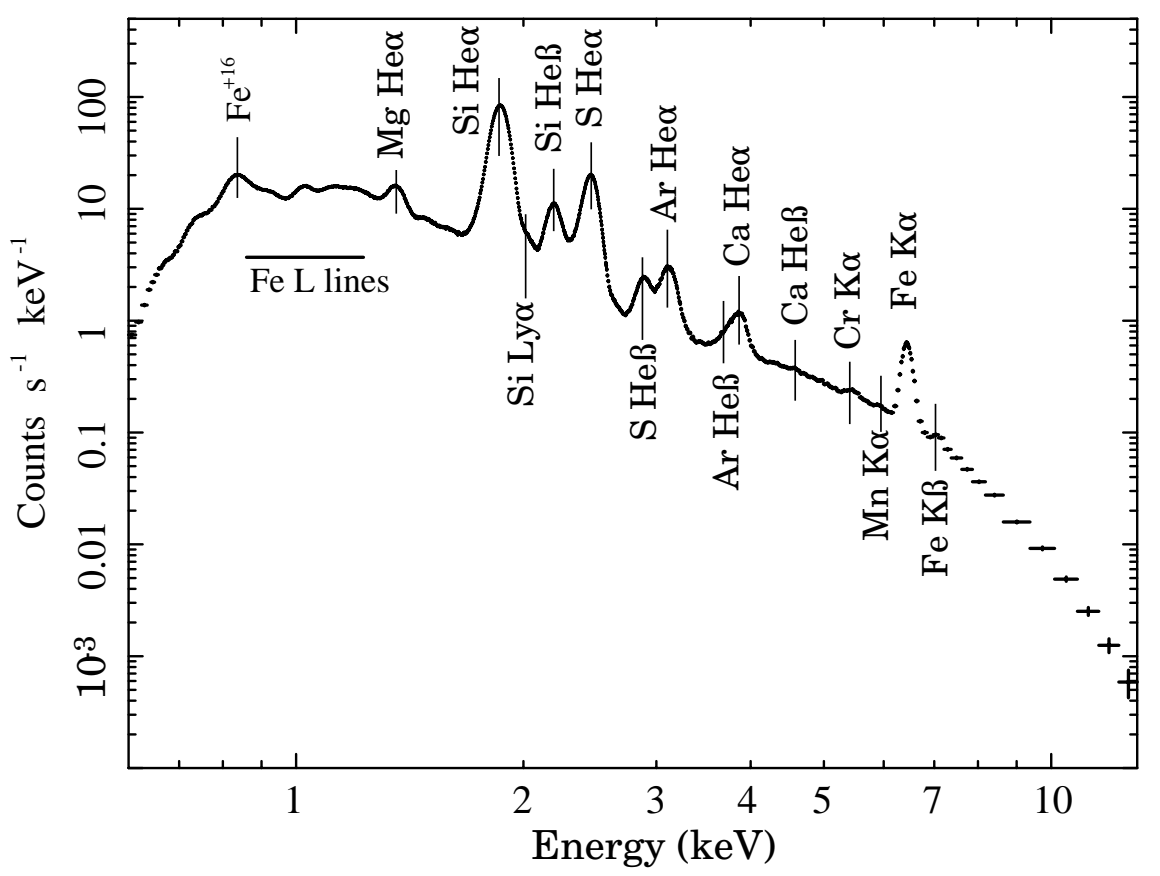

Fig. 2.- Background-subtracted XIS FI (XIS $0+2+3$ ) spectrum of the entire SNR. The important lines are marked. He $\alpha, \mathrm{He} \beta$, and Ly $\alpha$ indicate emission lines of $n=2 \rightarrow n=1$ transition in He-like ion, $1 s 3 p \rightarrow 1 s^{2}$ transition in He-like ion, and $n=2 \rightarrow n=1$ transition in H-like ion. 

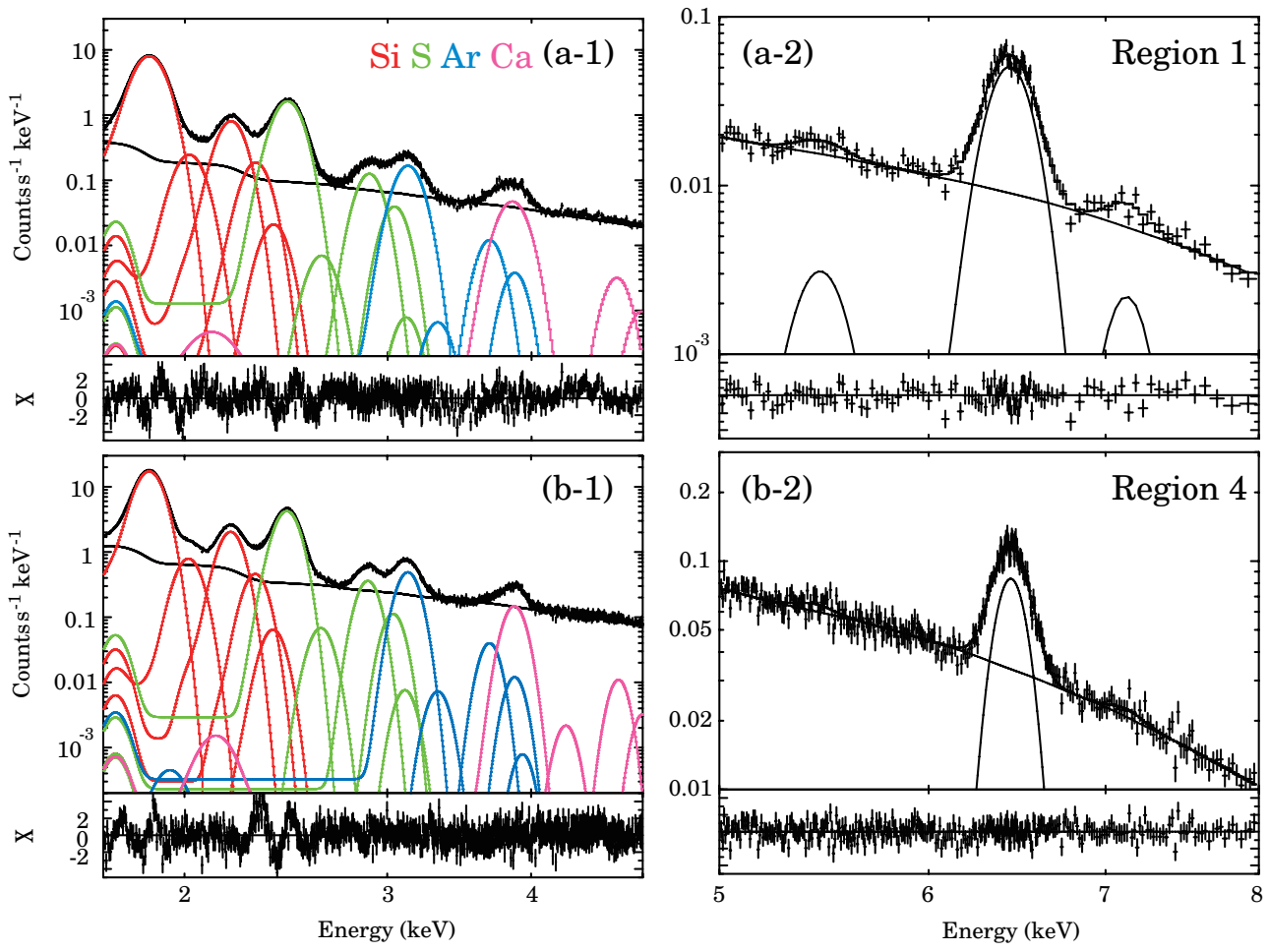

Fig. 3.- Background-subtracted $1.7-5 \mathrm{keV}$ (left) and 5-8 keV (right) band spectra extracted from region 1 (a) and 4 (b) indicated in Figure 1. The best-fit models are shown in solid lines and residuals are given in the bottom panels. 

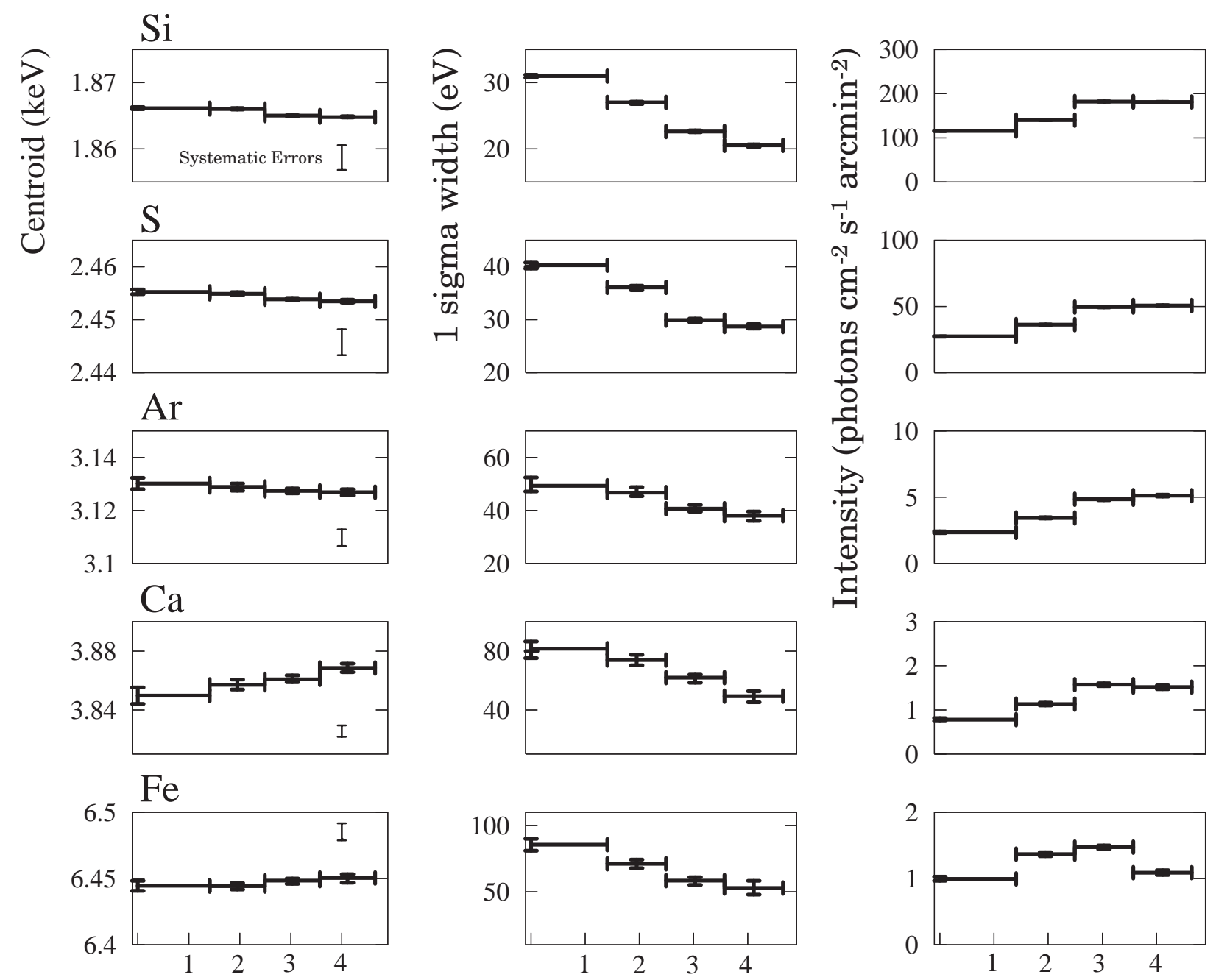

\section{Distance from center (arcmin)}

Fig. 4.- Radial dependence of the best-fit Gaussian parameters of $\mathrm{He} \alpha$ lines of $\mathrm{Si}, \mathrm{S}$, Ar, and Ca, and Fe K $\alpha$ blend. The centroid energies (left), the $1 \sigma$ widths (middle), and the intensities (right). The systematic errors from the intrachip gain variation at $90 \%$ confidence level are represented at the right corner in each left box. The statistical errors also represent $90 \%$ confidence level. 

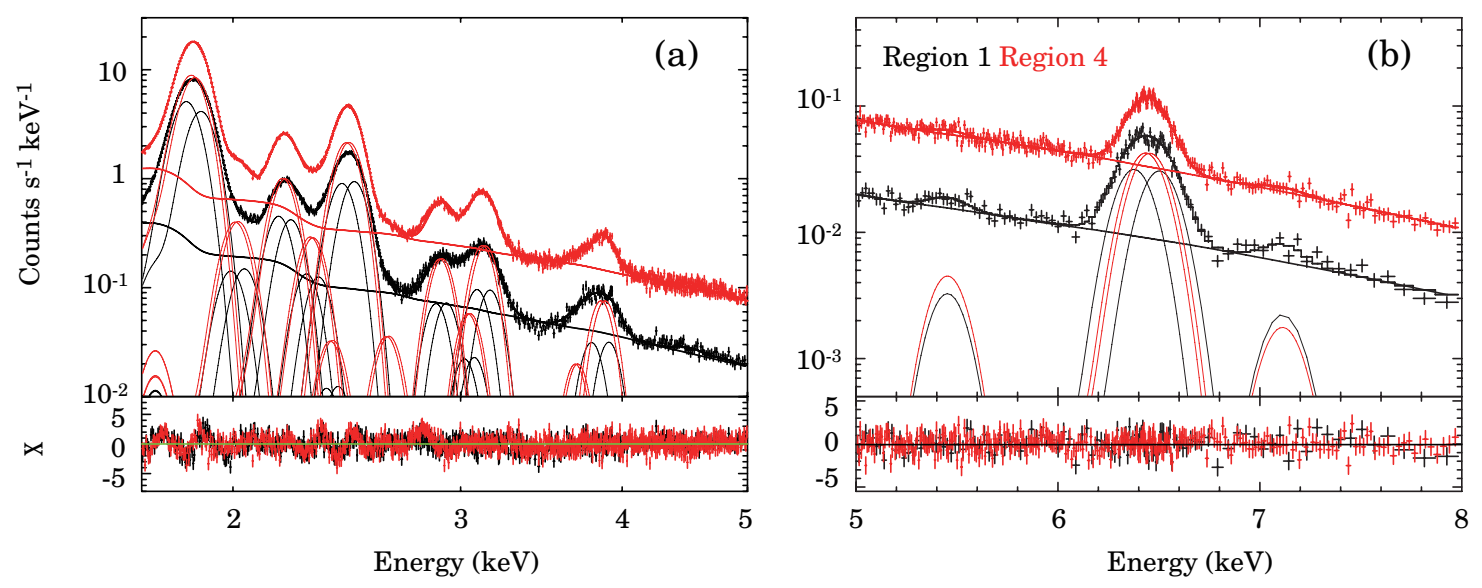

Fig. 5.- (a) $1.7-5 \mathrm{keV}$ and (b) 5-8 keV spectra of region 1 (black) and 4 (red) with the best-fit double Gaussian models.

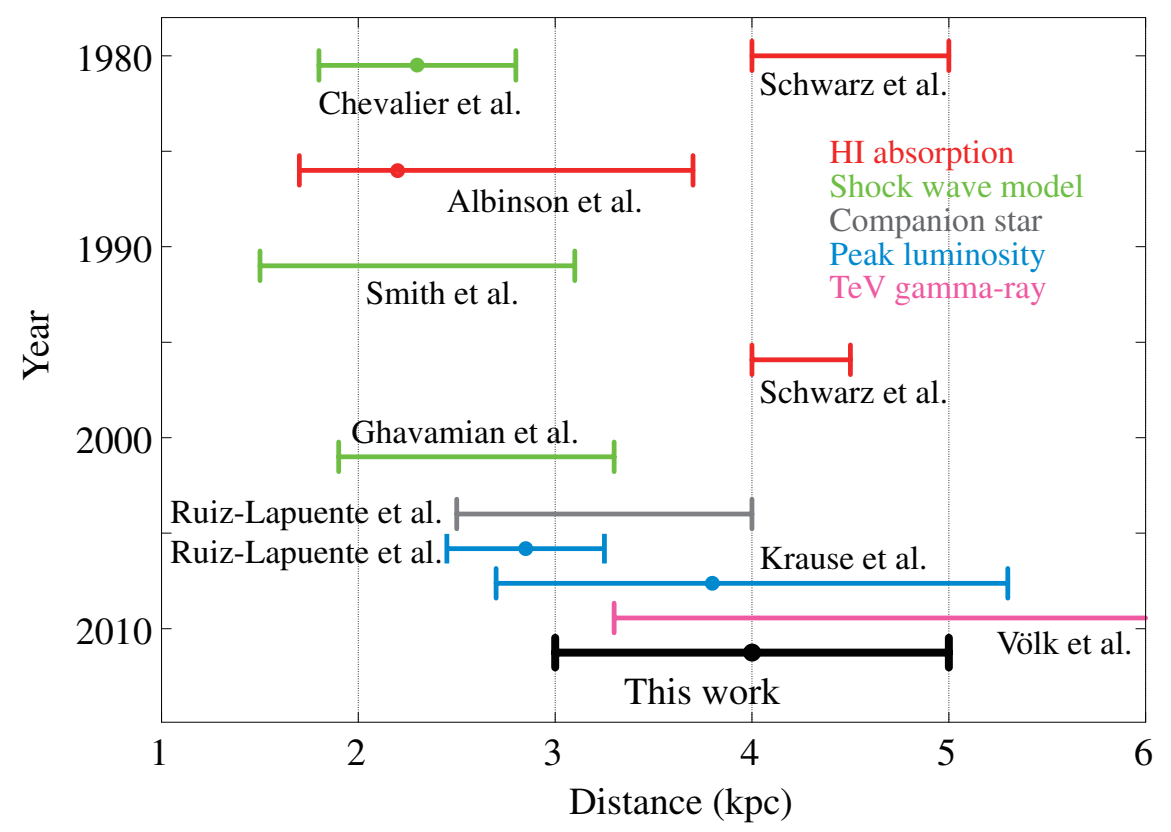

Fig. 6. - Estimates of the distance to Tycho's SNR. 


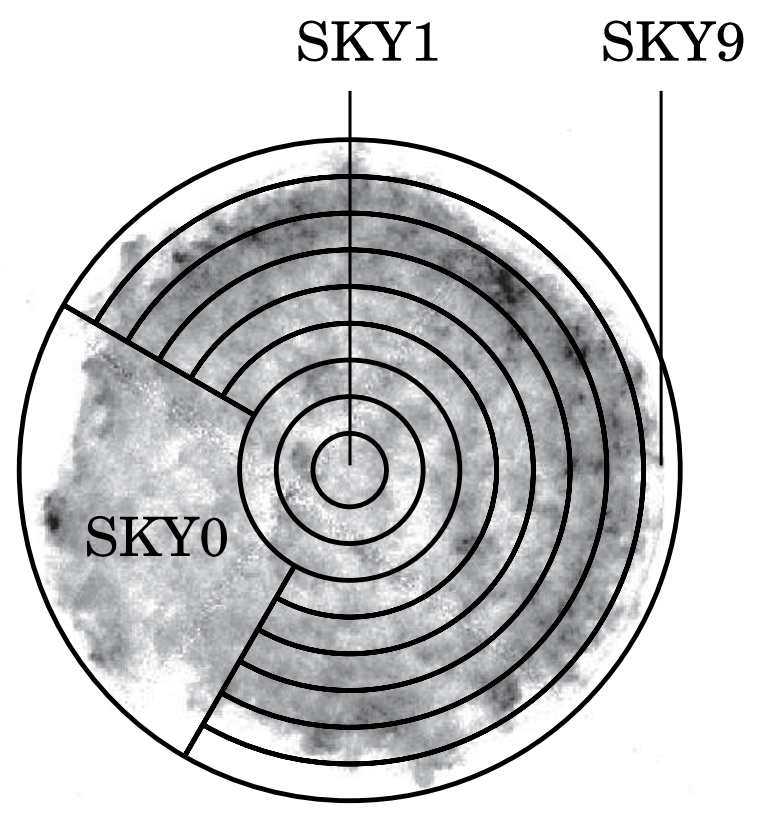

Fig. 7.- SKY regions defined in the plane of the sky. The underlying image was taken by Chandra. 
Table 1. Best-fit Gaussian parameters

\begin{tabular}{|c|c|c|c|c|c|c|}
\hline \multirow[b]{2}{*}{ Line } & \multicolumn{3}{|c|}{ Region 1} & \multicolumn{3}{|c|}{ Region 2} \\
\hline & $\begin{array}{c}\text { Centroid } \\
(\mathrm{keV})\end{array}$ & $\begin{array}{c}\text { Width }(1 \sigma) \\
(\mathrm{eV})\end{array}$ & Flux $^{a}$ & $\begin{array}{c}\text { Centroid } \\
(\mathrm{keV})\end{array}$ & $\begin{array}{c}\text { Width }(1 \sigma) \\
(\mathrm{eV})\end{array}$ & Flux $^{a}$ \\
\hline $\mathrm{Si} \operatorname{He} \alpha$ & $1.8661_{-0.0001}^{+0.0002}$ & $31.0 \pm 0.2$ & $115.7 \pm 0.3$ & $1.86603_{-0.00008}^{+0.00014}$ & $27.0_{-0.2}^{+0.1}$ & $140.3_{-0.301}^{+0.293}$ \\
\hline Si Ly $\alpha$ & $=\mathrm{He} \beta-0.178$ & $=\mathrm{He} \beta$ & $3.5 \pm 0.1$ & $=\mathrm{He} \beta-0.178$ & $=\operatorname{He} \beta$ & $4.94_{0.09}^{+0.11}$ \\
\hline $\mathrm{Si} \operatorname{He} \beta$ & $2.1972_{-0.0008}^{+0.0012}$ & $36 \pm 1$ & $10.4 \pm 0.1$ & $2.1962_{-0.0008}^{+0.0005}$ & $30.5_{-1}^{+0.6}$ & $13.26_{0.09}^{+0.11}$ \\
\hline $\mathrm{Si} \mathrm{He \gamma}$ & $=\mathrm{He} \beta+0.111$ & $=\mathrm{He} \beta$ & $(\mathrm{He} \gamma / \mathrm{He} \beta=0.3)$ & $=\mathrm{He} \beta+0.111$ & $=\operatorname{He} \beta$ & $(\mathrm{He} \gamma / \mathrm{He} \beta=0.3)$ \\
\hline $\mathrm{Si} \operatorname{Ly} \beta$ & $=\mathrm{He} \beta+0.193$ & $=\mathrm{He} \beta$ & $(\operatorname{Ly} \beta / \operatorname{Ly} \alpha=0.1)$ & $=\mathrm{He} \beta+0.193$ & $=\mathrm{He} \beta$ & $(\operatorname{Ly} \beta / \operatorname{Ly} \alpha=0.1)$ \\
\hline $\mathrm{S} \mathrm{He} \alpha$ & $2.4553_{-0.0004}^{+0.0005}$ & $40.3_{-0.6}^{+0.5}$ & $27.4 \pm 0.2$ & $2.4549 \pm 0.0003$ & $36.1_{-0.6}^{+0.3}$ & $36.3 \pm 0.2$ \\
\hline $\mathrm{S} \operatorname{Ly} \alpha$ & $=\mathrm{He} \beta-0.263$ & $=\operatorname{He} \beta$ & $0.18 \pm 0.06$ & $=\mathrm{He} \beta-0.263$ & $=\operatorname{He} \beta$ & $0.395_{0.046}^{+0.056}$ \\
\hline $\mathrm{S} \mathrm{He} \beta$ & $2.899 \pm 0.003$ & $59_{-3}^{+5}$ & $2.05 \pm 0.07$ & $2.887 \pm 0.002$ & $44_{-3}^{+2}$ & $2.58 \pm 0.05$ \\
\hline $\mathrm{S} \mathrm{He} \gamma$ & $=\mathrm{He} \beta+0.149$ & $=\mathrm{He} \beta$ & $(\mathrm{He} \gamma / \mathrm{He} \beta=0.3)$ & $=\mathrm{He} \beta+0.149$ & $=\mathrm{He} \beta$ & $(\mathrm{He} \gamma / \mathrm{He} \beta=0.3)$ \\
\hline $\mathrm{S} \operatorname{Ly} \beta$ & $=\mathrm{He} \beta+0.222$ & $=\mathrm{He} \beta$ & $(\operatorname{Ly} \beta / \operatorname{Ly} \alpha=0.1)$ & $=\mathrm{He} \beta+0.222$ & $=\mathrm{He} \beta$ & $(\operatorname{Ly} \beta / \operatorname{Ly} \alpha=0.1)$ \\
\hline $\operatorname{Ar~} \operatorname{He} \alpha$ & $3.130 \pm 0.002$ & $49_{-2}^{+3}$ & $2.35_{-0.06}^{+0.07}$ & $3.129 \pm 0.001$ & $47_{-1}^{+2}$ & $3.44_{-0.05}^{+0.06}$ \\
\hline $\operatorname{Ar} \operatorname{Ly} \alpha$ & $=\mathrm{He} \alpha+0.209$ & $=\mathrm{He} \alpha$ & $\mathrm{b}$ & $=\mathrm{He} \alpha+0.209$ & $=\operatorname{He} \alpha$ & $\mathrm{b}$ \\
\hline $\operatorname{Ar} \mathrm{He} \beta$ & $=\mathrm{He} \alpha+0.561$ & $=\operatorname{He} \alpha$ & $\mathrm{c}$ & $=\operatorname{He} \alpha+0.561$ & $=\operatorname{He} \alpha$ & $\mathrm{c}$ \\
\hline Ar $\mathrm{He} \gamma$ & $=\mathrm{He} \alpha+0.751$ & $=\mathrm{He} \alpha$ & $(\mathrm{He} \gamma / \mathrm{He} \beta=0.3)$ & $=\mathrm{He} \alpha+0.751$ & $=\mathrm{He} \alpha$ & $(\mathrm{He} \gamma / \mathrm{He} \beta=0.3)$ \\
\hline $\operatorname{Ar} \operatorname{Ly} \beta$ & $=\mathrm{He} \alpha+0.812$ & $=\operatorname{He} \alpha$ & $(\operatorname{Ly} \beta / \operatorname{Ly} \alpha=0.1)$ & $=\mathrm{He} \alpha+0.812$ & $=\operatorname{He} \alpha$ & $(\operatorname{Ly} \beta / \operatorname{Ly} \alpha=0.1)$ \\
\hline $\mathrm{Ca} \operatorname{He} \alpha$ & $3.850 \pm 0.006$ & $82_{-6}^{+5}$ & $0.78 \pm 0.03$ & $3.857_{-0.003}^{+0.004}$ & $74 \pm 4$ & $1.13 \pm 0.03$ \\
\hline $\mathrm{Ca} \operatorname{Ly} \alpha$ & $=\mathrm{He} \alpha+0.220$ & $=\operatorname{He} \alpha$ & $\mathrm{b}$ & $=\operatorname{He} \alpha+0.220$ & $=\operatorname{He} \alpha$ & $\mathrm{b}$ \\
\hline $\mathrm{Ca} \mathrm{He} \beta$ & $=\operatorname{He} \alpha+0.654$ & $=\operatorname{He} \alpha$ & c & $=\operatorname{He} \alpha+0.654$ & $=\operatorname{He} \alpha$ & c \\
\hline $\mathrm{Ca} \mathrm{He} \gamma$ & $=\mathrm{He} \alpha+0.938$ & $=\operatorname{He} \alpha$ & $(\mathrm{He} \gamma / \mathrm{He} \beta=0.3)$ & $=\operatorname{He} \alpha+0.938$ & $=\operatorname{He} \alpha$ & $(\mathrm{He} \gamma / \mathrm{He} \beta=0.3)$ \\
\hline $\mathrm{Ca} \operatorname{Ly} \beta$ & $=\mathrm{He} \alpha+0.979$ & $=\operatorname{He} \alpha$ & $(\operatorname{Ly} \beta / \operatorname{Ly} \alpha=0.1)$ & $=\mathrm{He} \alpha+0.979$ & $=\operatorname{He} \alpha$ & $(\operatorname{Ly} \beta / \operatorname{Ly} \alpha=0.1)$ \\
\hline $\mathrm{Cr} \mathrm{K \alpha}$ & $5.46 \pm 0.04$ & $=\mathrm{Fe} \mathrm{K} \alpha$ & $0.05 \pm 0.02$ & $5.51_{-0.05}^{+0.04}$ & $=\mathrm{Fe} \mathrm{K} \alpha$ & $0.05 \pm 0.02$ \\
\hline $\mathrm{Fe} K \alpha$ & $6.445 \pm 0.004$ & $86 \pm 4$ & $1.00 \pm 0.03$ & $6.444_{-0.003}^{+0.002}$ & $71 \pm 3$ & $1.37 \pm 0.03$ \\
\hline $\mathrm{Fe} \mathrm{K} \beta$ & $7.14 \pm 0.05$ & $=\mathrm{Fe} \mathrm{K} \alpha$ & $0.05 \pm 0.02$ & $\begin{array}{l}7.11 \pm 0.02 \\
\end{array}$ & $=\mathrm{Fe} \mathrm{K} \alpha$ & $0.08_{-0.01}^{+0.02}$ \\
\hline \multirow[b]{2}{*}{ Line } & \multicolumn{3}{|c|}{ Region 3} & \multicolumn{3}{|c|}{ Region 4} \\
\hline & $\begin{array}{c}\text { Centroid } \\
(\mathrm{keV})\end{array}$ & $\begin{array}{c}\text { Width }(1 \sigma) \\
(\mathrm{eV})\end{array}$ & Flux $^{a}$ & $\begin{array}{c}\text { Centroid } \\
(\mathrm{keV})\end{array}$ & $\begin{array}{c}\text { Width }(1 \sigma) \\
(\mathrm{eV})\end{array}$ & Flux $^{\mathrm{a}}$ \\
\hline $\mathrm{Si} \operatorname{He} \alpha$ & $1.86504_{-0.00011}^{+0.00006}$ & $22.6_{-0.1}^{+0.2}$ & $182.4_{-0.2}^{+0.4}$ & $1.86481_{-0.00007}^{+0.00015}$ & $20.5_{-0.2}^{+0.1}$ & $181.2_{-0.4}^{+0.3}$ \\
\hline Si Ly $\alpha$ & $=\mathrm{He} \beta-0.178$ & $=\operatorname{He} \beta$ & $7.28_{-0.13}^{+0.09}$ & $=\mathrm{He} \beta-0.178$ & $=\mathrm{He} \beta$ & $7.5 \pm 0.1$ \\
\hline $\mathrm{Si} \operatorname{He} \beta$ & $2.1945_{-0.0007}^{+0.0003}$ & $23.6_{-0.7}^{+0.6}$ & $17.69_{-0.07}^{+0.14}$ & $2.1935_{-0.0006}^{+0.0005}$ & $21.1_{-1.2}^{+0.5}$ & $18.0 \pm 0.2$ \\
\hline $\mathrm{Si} \mathrm{He \gamma}$ & $=\mathrm{He} \beta+0.111$ & $=\operatorname{He} \beta$ & $(\mathrm{He} \gamma / \mathrm{He} \beta=0.3)$ & $=\mathrm{He} \beta+0.111$ & $=\operatorname{He} \beta$ & $(\mathrm{He} \gamma / \mathrm{He} \beta=0.3)$ \\
\hline $\mathrm{Si} \operatorname{Ly} \beta$ & $=\mathrm{He} \beta+0.193$ & $=\mathrm{He} \beta$ & $(\operatorname{Ly} \beta / \operatorname{Ly} \alpha=0.1)$ & $=\mathrm{He} \beta+0.193$ & $=\operatorname{He} \beta$ & $(\operatorname{Ly} \beta / \operatorname{Ly} \alpha=0.1)$ \\
\hline $\mathrm{S} \mathrm{He} \alpha$ & $2.4539 \pm 0.0002$ & $29.9_{-0.4}^{+0.3}$ & $49.6_{-0.2}^{+0.1}$ & $2.4535_{-0.0002}^{0.0003}$ & $28.7_{-0.4}^{+0.5}$ & $50.7_{-0.2}^{+0.3}$ \\
\hline $\mathrm{S} \operatorname{Ly} \alpha$ & $=\mathrm{He} \beta-0.263$ & $=\operatorname{He} \beta$ & $0.75_{-0.05}^{+0.06}$ & $=\mathrm{He} \beta-0.263$ & $=\operatorname{He} \beta$ & $0.76_{-0.06}^{+0.09}$ \\
\hline $\mathrm{S} \mathrm{He} \beta$ & $2.888_{-0.001}^{+0.002}$ & $34_{-2}^{+1}$ & $3.54_{-0.04}^{+0.06}$ & $2.89_{-0.01}^{+0.02}$ & $35_{-0.3}^{+0.2}$ & $3.74_{-0.06}^{+0.12}$ \\
\hline $\mathrm{S} \mathrm{He} \gamma$ & $=\mathrm{He} \beta+0.149$ & $=\mathrm{He} \beta$ & $(\mathrm{He} \gamma / \mathrm{He} \beta=0.3)$ & $=\mathrm{He} \beta+0.149$ & $=\mathrm{He} \beta$ & $(\mathrm{He} \gamma / \mathrm{He} \beta=0.3)$ \\
\hline $\mathrm{S} \mathrm{Ly} \beta$ & $=\mathrm{He} \beta+0.222$ & $=\mathrm{He} \beta$ & $(\operatorname{Ly} \beta / \operatorname{Ly} \alpha=0.1)$ & $=\mathrm{He} \beta+0.222$ & $=\mathrm{He} \beta$ & $(\operatorname{Ly} \beta / \operatorname{Ly} \alpha=0.1)$ \\
\hline $\operatorname{Ar} \operatorname{He} \alpha$ & $3.1273_{-0.0009}^{+0.0010}$ & $41_{-1}^{+1}$ & $4.84_{-0.06}^{+0.05}$ & $3.127 \pm 0.01$ & $38 \pm 3$ & $5.11_{-0.07}^{+0.08}$ \\
\hline $\operatorname{Ar} \operatorname{Ly} \alpha$ & $=\operatorname{He} \alpha+0.209$ & $=\operatorname{He} \alpha$ & $\mathrm{b}$ & $=\mathrm{He} \alpha+0.209$ & $=\mathrm{He} \alpha$ & $\mathrm{b}$ \\
\hline $\operatorname{Ar} \mathrm{He} \beta$ & $=\mathrm{He} \alpha+0.561$ & $=\mathrm{He} \alpha$ & $\mathrm{c}$ & $=\operatorname{He} \alpha+0.561$ & $=\mathrm{He} \alpha$ & $\mathrm{c}$ \\
\hline $\mathrm{Ar} \mathrm{He} \gamma$ & $=\operatorname{He} \alpha+0.751$ & $=\operatorname{He} \alpha$ & $(\mathrm{He} \gamma / \mathrm{He} \beta=0.3)$ & $=\mathrm{He} \alpha+0.751$ & $=\operatorname{He} \alpha$ & $(\mathrm{He} \gamma / \mathrm{He} \beta=0.3)$ \\
\hline $\operatorname{Ar} \operatorname{Ly} \beta$ & $=$ He $\alpha+0.812$ & $=\mathrm{He} \alpha$ & $(\operatorname{Ly} \beta / \operatorname{Ly} \alpha=0.1)$ & $=\operatorname{He} \alpha+0.812$ & $=\mathrm{He} \alpha$ & $(\operatorname{Ly} \beta / \operatorname{Ly} \alpha=0.1)$ \\
\hline $\mathrm{Ca} \operatorname{He} \alpha$ & $3.8612_{-0.002}^{+0.003}$ & $62_{-3}^{+2}$ & $1.57_{-0.04}^{+0.03}$ & $3.868 \pm 0.03$ & $49_{-4}^{+3}$ & $1.52_{-0.05}^{+0.04}$ \\
\hline Ca Ly $\alpha$ & $=\mathrm{He} \alpha+0.220$ & $=\mathrm{He} \alpha$ & $\mathrm{b}$ & $=\mathrm{He} \alpha+0.220$ & $=\operatorname{He} \alpha$ & $\mathrm{b}$ \\
\hline $\mathrm{Ca} \mathrm{He} \beta$ & $=\mathrm{He} \alpha+0.654$ & $=\mathrm{He} \alpha$ & c & $=\mathrm{He} \alpha+0.654$ & $=\mathrm{He} \alpha$ & c \\
\hline $\mathrm{Ca} \mathrm{He} \gamma$ & $=\mathrm{He} \alpha+0.938$ & $=\mathrm{He} \alpha$ & $(\mathrm{He} \gamma / \mathrm{He} \beta=0.3)$ & $=\mathrm{He} \alpha+0.938$ & $=\mathrm{He} \alpha$ & $(\mathrm{He} \gamma / \mathrm{He} \beta=0.3)$ \\
\hline
\end{tabular}


Table 1-Continued

\begin{tabular}{ccccccc}
$\mathrm{Ca} \operatorname{Ly} \beta$ & $=\mathrm{He} \alpha+0.979$ & $=\mathrm{He} \alpha$ & $(\mathrm{Ly} \beta / \mathrm{Ly} \alpha=0.1)$ & $=\mathrm{He} \alpha+0.979$ & $=\mathrm{He} \alpha$ & $(\mathrm{Ly} \beta / \operatorname{Ly} \alpha=0.1)$ \\
\hline $\mathrm{Cr} \mathrm{K} \alpha$ & $5.48 \pm 0.03$ & $=\mathrm{Fe} \mathrm{K} \alpha$ & $0.06 \pm 0.02$ & $5.44_{-0.04}^{+0.05}$ & $=\mathrm{Fe} \mathrm{K} \alpha$ & $0.05 \pm 0.03$ \\
$\mathrm{Fe} \mathrm{K} \alpha$ & $6.448 \pm+0.002$ & $58 \pm 3$ & $1.47 \pm 0.03$ & $6.450_{-0.004}^{+0.003}$ & $53_{-5}^{+6}$ & $1.09_{-0.03}^{+0.04}$ \\
$\mathrm{Fe} \mathrm{K} \beta$ & $7.12 \pm 0.03$ & $=\mathrm{Fe} \mathrm{K} \alpha$ & $0.05_{-0.02}^{+0.01}$ & $7.13 \pm 0.09$ & $=\mathrm{Fe} \mathrm{K} \alpha$ & $0.03 \pm 0.02$ \\
\hline
\end{tabular}

Note. - Errors indicate the $90 \%$ confidence limits.

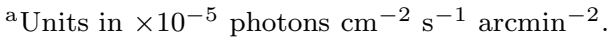

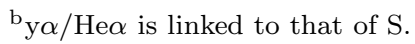

${ }^{\mathrm{c}} \mathrm{He} \beta / \mathrm{He} \alpha$ is linked to that of $\mathrm{S}$.

Table 2: Correction factors $C_{i}$ defined in Equation 2

\begin{tabular}{lcc}
\hline \hline & IMEs & Fe \\
\hline Region 1 & 0.748 & 0.712 \\
Region 2 & 0.578 & 0.530 \\
Region 3 & 0.315 & 0.262 \\
Region 4 & 0.170 & 0.120 \\
\hline
\end{tabular}

Table 3: Best-fit parameters of the double Gaussian model

\begin{tabular}{ccccccc}
\hline \hline & $\begin{array}{c}\text { Width } \\
(\mathrm{eV})\end{array}$ & $\begin{array}{c}E_{\text {red }} \\
(\mathrm{keV})\end{array}$ & $\begin{array}{c}E_{\text {blue }} \\
(\mathrm{keV})\end{array}$ & $\begin{array}{c}2 \delta E_{1} \\
(\mathrm{eV})\end{array}$ & $\begin{array}{c}v_{\perp 1} \\
\left(\mathrm{~km} \mathrm{~s}^{-1}\right)\end{array}$ & $\begin{array}{c}v_{\text {exp }} \\
\left(\mathrm{km} \mathrm{s}^{-1}\right)\end{array}$ \\
\hline Si He$\alpha$ & $20.16_{-0.09}^{+0.11}$ & $1.8883_{-0.0001}^{+0.0002}$ & $1.8443 \pm 0.0002$ & $44.0_{-0.2}^{+0.3}$ & $3540 \pm 20$ & $4730_{-20}^{+30}$ \\
$\mathrm{Si} \mathrm{He} \beta$ & $17.8_{-0.7}^{+0.4}$ & $2.1685_{-0.0012}^{+0.0007}$ & $2.2194_{-0.0006}^{+0.00014}$ & $51_{-2}^{+1}$ & $3480_{-100}^{+90}$ & $4700 \pm 100$ \\
$\mathrm{~S} \mathrm{He} \alpha$ & $26.6_{-0.3}^{+0.2}$ & $2.4270_{-0.0005}^{+0.0004}$ & $2.4841_{-0.0003}^{+0.0003}$ & $57.1 \pm 0.6$ & $3490 \pm 40$ & $4660 \pm 50$ \\
$\mathrm{Ar} \mathrm{He} \alpha$ & $34.6_{-0.7}^{+1.4}$ & $3.089 \pm 0.002$ & $3.164 \pm+0.002$ & $74 \pm 3$ & $3600 \pm 100$ & $4800 \pm 200$ \\
\hline Fe K $\alpha$ & $55 \pm 3$ & $6.507_{-0.007}^{+0.005}$ & $6.383_{-0.005}^{+0.007}$ & $124 \pm 8$ & $2900 \pm 200$ & $4000 \pm 300$ \\
\hline
\end{tabular}

Note. - Errors indicate the $90 \%$ confidence limits.

${ }^{a}$ Best fit values of region 1 .

Table 4: Calculated $\bar{A}_{j}$ described in Equation A7

\begin{tabular}{lcccccccccc}
\hline \hline & SKY0 & SKY1 & SKY2 & SKY3 & SKY4 & SKY5 & SKY6 & SKY7 & SKY8 & SKY9 \\
\hline Si Shell* $^{*}$ & 0.455 & 0.995 & 0.973 & 0.927 & 0.854 & 0.744 & 0.573 & 0.138 & 0 & 0 \\
Fe Shell $^{\dagger}$ & 0.447 & 0.994 & 0.968 & 0.914 & 0.827 & 0.693 & 0.450 & 0 & 0 & 0 \\
\hline
\end{tabular}

${ }^{*} r_{\mathrm{sh}}=190^{\prime \prime}-220^{\prime \prime}$
${ }^{\dagger} r_{\mathrm{sh}}=180^{\prime \prime}-200^{\prime \prime}$ 
Table 5: Results of the simulation: fraction of the photons $F_{i j}$ detected in REG $i$ to those originated from SKY $j$

\begin{tabular}{|c|c|c|c|c|c|c|c|c|c|c|}
\hline \multicolumn{11}{|c|}{ Si and $\mathrm{S}$} \\
\hline & SKY 0 & SKY 1 & SKY 2 & SKY 3 & SKY 4 & SKY 5 & SKY 6 & SKY 7 & SKY 8 & SKY 9 \\
\hline Region 1 & 0.100 & 0.068 & 0.190 & 0.207 & 0.152 & 0.098 & 0.081 & 0.071 & 0.030 & 0.003 \\
\hline Region 2 & 0.047 & 0.010 & 0.034 & 0.087 & 0.171 & 0.203 & 0.195 & 0.175 & 0.071 & 0.006 \\
\hline Region 3 & 0.033 & 0.002 & 0.007 & 0.016 & 0.038 & 0.089 & 0.220 & 0.383 & 0.196 & 0.017 \\
\hline Region 4 & 0.031 & 0.001 & 0.003 & 0.007 & 0.017 & 0.037 & 0.104 & 0.325 & 0.393 & 0.082 \\
\hline \multicolumn{11}{|c|}{$\mathrm{Fe}$} \\
\hline & SKY 0 & SKY 1 & SKY 2 & SKY 3 & SKY 4 & SKY 5 & SKY 6 & SKY 7 & SKY 8 & SKY 9 \\
\hline Region 1 & 0.105 & 0.064 & 0.178 & 0.194 & 0.154 & 0.113 & 0.099 & 0.062 & 0.024 & 0.006 \\
\hline Region 2 & 0.050 & 0.009 & 0.032 & 0.079 & 0.164 & 0.223 & 0.235 & 0.143 & 0.054 & 0.013 \\
\hline Region 3 & 0.037 & 0.002 & 0.006 & 0.016 & 0.039 & 0.100 & 0.271 & 0.335 & 0.160 & 0.036 \\
\hline Region 4 & 0.036 & 0.001 & 0.003 & 0.006 & 0.016 & 0.040 & 0.120 & 0.261 & 0.341 & 0.176 \\
\hline
\end{tabular}

